\title{
Protocol with an unlisted collaborator
}

Dr. Orin Chambers, a neurophysiologist at Great Eastern University, was collaborating with Dr. Michael Bryant, a physics professor at nearby Milford University. Chambers and Bryant were jointly developing a perfluorocarbon-based blood substitute (more correctly called an oxygen therapeutic) that was enclosed in a unique nanoparticle for delivery to injured tissues. The nanoparticle enclosed both the blood substitute and a lytic agent to help dissolve intravascular thrombi. All of their preliminary work was conducted in cell cultures. There were no indications of cytotoxicity, the product functioned in vitro as expected, and the two researchers were anxious to begin preliminary animal studies for safety and efficacy.

The model chosen was an outbred rat because there was a large amount of published knowledge about the anatomy and physiology of the rat cerebrovascular system and Chambers had extensive experience working with rat stroke models. The protocol was submitted to the Great Eastern IACUC with Chambers as the principal investigator. Bryant was not listed on the protocol because he would not be involved in any way with using live animals.

The protocol was approved, and soon afterwards, Chambers sent a request to the IACUC office to give Bryant access to the animal facility so that he could use the necropsy room to collect tissues for electron microscopy as soon as a rat was euthanized by Chambers' technician. Neither the IACUC office nor the IACUC chairperson had ever received such a request, and so they asked the attending veterinarian for an opinion. The veterinarian desired to be helpful, but she was somewhat concerned about allowing a person who was not listed on an IACUC-approved protocol to enter the animal facility and obtain tissues from a faculty member's animal.

What is your opinion? Does Chambers' request present any concerns for Great Eastern University? How do you think Chambers' request should be handled?

\section{RESPONSE}

\section{Add Bryant to the protocol}

\author{
Maggie Struck Behnke, DVM, DACLAM, \\ Brooke Bloomberg, DVM, MPH, MS \& \\ Sally Olson, DVM
}

The need for Bryant to be present in the vivarium to collect samples is not clearly justified in this scenario. Perhaps facility access for this collaborator is a matter of convenience for the research team rather than a scientific imperative. But denying animal facility access to a research team member would not promote a welcoming environment for investigators at Great Eastern, and we wouldn't recommend that option. Although our ultimate goal would be to grant Chambers' request, we share the veterinarian's concerns about a person who is not a university employee and not included on an IACUC-approved protocol working in the animal facility. We suggest that Bryant be included on the protocol and that his inclusion be approved by the Great Eastern IACUC.

A number of issues come into focus when considering animal facility access for an individual who has not been through the IACUC protocol approval process: occupational health and safety risks, a possible lack of adequate training in animal care and use and the potential for noncompliance to occur.

While working in the vivarium, Bryant will be exposed to potential health risks such as animal allergens, sharp instruments and nanoparticles containing lytic agents (the experimental article). Although these health risks might be minimal, they should be addressed by an occupational health and safety program (OHSP) to assure that the institution is providing a safe and healthy work environment, as recommended in the Guide for the Care and Use of Laboratory Animals ${ }^{1}$. Even though participation in an OHSP is not limited to individuals listed on IACUC-approved protocols, the approved inclusion of Bryant on the protocol would identify and associate him with the health risks mentioned above, triggering his participation in Great Eastern's OHSP.

In addition to providing a safe and healthy work environment, Great Eastern University has a responsibility to ensure that everyone on the research team (including a visiting scientist) has the necessary knowledge and expertise for the specific animal procedures proposed and the species used ${ }^{1,2}$. Bryant will not be handling live animals but will be in close proximity to live animals and will be actively collecting tissue samples for his research. Training about the ethics of animal use, animal care and use legislation, methods for reporting welfare concerns and occupational health and safety risks are all directly related to Bryant's role in the project ${ }^{1}$. It benefits both Great Eastern University and Bryant to require his training in these subjects.

The attending veterinarian could surely address occupational health and safety risks and training issues for Bryant outside of protocol approval. But this option ignores a potential opportunity to improve research team efficiency and introduces the potential for noncompliance. With his inclusion on the protocol, Bryant's role would not be limited to contact with animal tissues and carcasses but could also carry out procedures using live animals if the need arose. Without his inclusion on the protocol, if Bryant had even minimal contact with live animals at Great Eastern, it would be an instance of noncompliance.

Because Bryant's role in this project is limited to post mortem tissue collection, he 